\title{
On Co Distributive Pair and Dually Co Distributive Pair in a Lattice
}

\author{
K.V.R. Srinivas, Professor and R. Nanda kumar \\ Asst. Professor in Mathematics, Regency Institute of Technology, Adavipolam, Yanam - 533464. \\ U.T.of Pondicherry, near kakinada.
}

Abstract: In this paper we have obtained some interesting results on Co distributive pair in lattices. We also obtained some results on dually Co distributive pair in general lattice.

Key Words: Co distributive pair, Dually co distributive, d-prime element, Dually d-prime element, d-prime Ideals, Dually d-prime element.

\section{Introduction}

In this paper we have defined some definitions like Co distributive pair, d-meet irreducible element, $d$ prime element of a lattice ' $\mathrm{L}$ ', d-prime is transformed to dually co distributive pair, dually d-prime ideals, dually d-prime element.

Using above definitions we have achieved some theorems [like,(4) If $(\mathrm{x}, \mathrm{y})$ is dually co distributive then for any $a \in L,(x \wedge a),(y \wedge a)$ is also dually co distributive.(6) Relation between dually d-prime ideal with

(1) distributive (2) Standard (3) Neutral] and result(s), If 'a' is dually d-prime element $\Leftrightarrow$ [a) is dually d-prime and $(x \wedge a),(y \wedge a)$ is dually co distributive pair. Also we have obtained some of the most important theorems, (8) If $(a, b)$ and $(b, c)$ are dually codistributive, then $(a \wedge c, b)$ is also dually co distributive and (9) Suppouse $I$ is a sublattice of $L$ and $m_{a}, a \in I$, and $m_{a}$ is an ideal of $I$,minimal w.r.to the property of containing ' $a$ ', then there is a d-prime ideal ' $p$ ' of $L \ni P \cap I=m_{a}$ which is followed by lemma, If ' $L$ ' is any lattice, then every dually d-meet irreducible element is dually d-prime.

AMS(2000) Subject Classification :- 20M10

\section{In the first part of this paper we start with the following preliminaries}

Def (1):- Co distributive pair : Let ' $\mathrm{L}$ ' be a lattice, $\mathrm{x}, \mathrm{y} \in \mathrm{L}$, then $(\mathrm{x}, \mathrm{y})$ is said to be codistributive, if $(\mathrm{x} \wedge \mathrm{y}) \vee \mathrm{z}=(\mathrm{x} \vee \mathrm{z}) \wedge(\mathrm{y} \vee \mathrm{z}) \forall \mathrm{z} \in \mathrm{L}$.

Def (2):- d-meet irreducible element : An element ' $a$ ' of lattice ' $L$ ' is called d-meet irreducible $\Leftrightarrow a=x \wedge y$ and $(\mathrm{x}, \mathrm{y})$ is codistributive $\Rightarrow$ either $\mathrm{a}=\mathrm{x}$ or $\mathrm{a}=\mathrm{y}$.

Def (3):- d-prime element of a lattice $L$ : An element ' $a$ ' of a lattice is $d$-prime $\Leftrightarrow a \geq x \wedge y$ and $(\mathrm{x}, \mathrm{y})$ is codistributive $\Rightarrow$ either $\mathrm{a} \geq \mathrm{x}$ or $\mathrm{a} \geq \mathrm{y}$.

Def (4):- d-prime Ideals : An Ideal 'I' of a lattice ' $L$ ' is called a d-prime Ideal if for any codistributive pair $(a, b) \in L^{2}$ with $a \wedge b \in I$ then $a \in I$ or $b \in I$.

Theorem (1) :- Connection between d-meet irreducible element of a lattice ' $L$ ' with either distributive/Standard/Neutral.

Proof :- Let 'a' be a d-meet irreducible element, Also let $(x, y)$ be co distributive with $a=x \wedge y$. Claim:- (i) ' $a$ ' is distributive, i.e, $a \vee(x \wedge y)=(a \vee x) \wedge(a \vee y)$

Consider, $a \vee(x \wedge y)=a \vee(a)=a$.

Also, $(a \vee x) \wedge(a \vee y)=((x \wedge y) \vee x) \wedge((x \wedge y) \vee y)=x \wedge y=a$.

Converse :- If ' $a$ ' is distributive then ' $a$ ' is a d-meet irreducible element.

Consider,

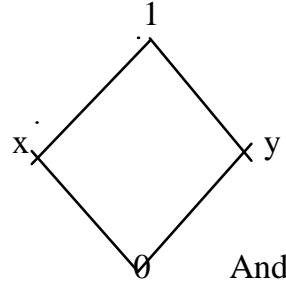

$\mathrm{a} \wedge \mathrm{b}=0, \mathrm{a} \vee \mathrm{b}=1$.

Since, $a \vee(x \wedge y)=(a \vee x) \wedge(a \vee y)$,

Let $x=1, y=b$, then $a \vee(1 \wedge b)=a \vee b=1$. Also, $(a \vee 1) \wedge(a \vee b)=1 \wedge 1=1$.

And $\mathrm{a} \wedge \mathrm{b}=0 \Rightarrow(\mathrm{a}, \mathrm{b})$ is co distributive $\Rightarrow$ either $\mathrm{a}=0$ or $\mathrm{b}=0$.

(ii) ' $a$ ' is standard, i.e, $x \wedge(a \vee y)=(x \wedge a) \vee(x \wedge y)$.

Consider, $\mathrm{x} \wedge(\mathrm{a} \vee \mathrm{y})=\mathrm{x} \wedge((\mathrm{x} \wedge \mathrm{y}) \vee \mathrm{y})=\mathrm{x} \wedge \mathrm{y}=\mathrm{a}$.

Also, $(x \wedge a) \vee(x \wedge y)=(x \wedge a) \vee a=a$.

Converse :- If ' $a$ ' is standard, then ' $a$ ' is not $d$-meet irreducible element, because of the following 
example,

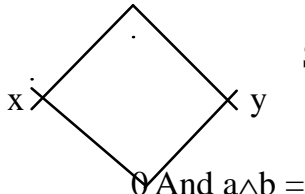

Since, $x \wedge(a \vee y)=(x \wedge a) \vee(x \wedge y)$,

Let $\mathrm{x}=1, \mathrm{y}=\mathrm{b}$, then $1 \wedge(\mathrm{a} \vee \mathrm{b})=1 \wedge 1=1$.

Also, $(x \wedge a) \vee(x \wedge y)=(1 \wedge a) \vee(1 \wedge b)=a \vee b=1$.

Theorem (2) :- Connection between d-prime element of a lattice ' $L$ ' with either distributive / Standard /

Neutral.

Proof :- Let ' $a$ ' is d-prime element, also let (x,y) be codistributive with $a \geq x \wedge y$.

Claim:- (i) ' $a$ ' is distributive, i.e, $a \vee(x \wedge y)=(a \vee x) \wedge(a \vee y)$.

Since, $a \geq x \wedge y \Rightarrow(x \wedge y) \vee a=a . \Rightarrow(x \vee a) \wedge(y \vee a)=a$., as $(x, y)$ is co distributive. $\Rightarrow$ ' $\mathrm{a}$ ' is distributive.

Converse of this need not be true, because of the following example,

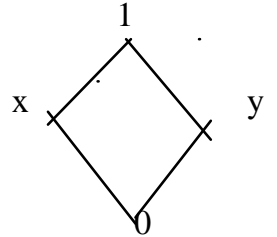

Since, $a \vee(x \wedge y)=(a \vee x) \wedge(a \vee y)$

$0 \vee(x \wedge y)=(0 \vee x) \wedge(0 \vee y)$

$0 \vee 0=\mathrm{x} \wedge \mathrm{y}$,

$0=0$.

$\therefore\{0\}$ is distributive.

Since, $0 \geq \mathrm{x} \wedge \mathrm{y}$ and (x,y) is codistributive, but, $0 \square \mathrm{x}$ and $0 \square \mathrm{y}$.

$\therefore$ Any distributive element need not be d-prime element.

(ii) ' $a$ ' is standard, i.e, $x \wedge(a \vee y)=(x \wedge a) \vee(x \wedge y)$.

Consider, $x \wedge(a \vee y)=(x \vee(x \wedge y)) \wedge(a \vee y)=(x \vee a) \wedge(y \vee a)=(x \wedge y) \wedge a=a \wedge a=a$.

Also, $(x \wedge a) \vee(x \wedge y)=(x \wedge a) \vee a=a$.

Converse of this need not be true, because of the following example,

1

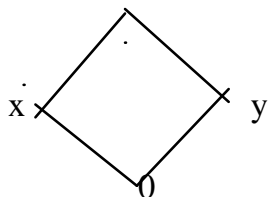

Since, $x \wedge(a \vee y)=(x \wedge a) \vee(x \wedge y)$, $\mathrm{x} \wedge(0 \vee \mathrm{y})=(\mathrm{x} \wedge 0) \vee(\mathrm{x} \wedge \mathrm{y})$

$\mathrm{x} \wedge \mathrm{y}=0 \vee 0$, $0=0$.

$\therefore\{0\}$ is standard.

Since, $0 \geq \mathrm{x} \wedge \mathrm{y}$ and (x,y) is co distributive, but, $0 \square \mathrm{x}$ and $0 \square \mathrm{y}$.

$\therefore$ Any Standard element need not be d-prime element.

\section{In the second part of the paper we start with the following preliminaries}

Def (1):- Dually co distributive : Let ' $\mathrm{L}$ ' be a lattice and $(\mathrm{x}, \mathrm{y}) \in \mathrm{L}^{2}$, then the pair $(\mathrm{x}, \mathrm{y})$ is said to be dually co distributive, if $(\mathrm{x} \vee \mathrm{y}) \wedge \mathrm{z}=(\mathrm{x} \wedge \mathrm{z}) \vee(\mathrm{y} \wedge \mathrm{z}) \forall \mathrm{z} \in \mathrm{L}$.

Def (2):- Dually d-prime Ideal : An Ideal $P$ of $L$ is called a dually d-prime Ideal if $(x, y) \in L^{2}$ with $(x \vee y) \in P$. $\Rightarrow \mathrm{x} \in \mathrm{P}$ and $\mathrm{y} \in \mathrm{P}$ for any codistributive pair $(\mathrm{x}, \mathrm{y})$ in $\mathrm{L}^{2}$.

Def (3):- Dually d-prime element : An element ' $a$ ' of a lattice ' $L$ ' is dually d-prime $\Leftrightarrow a \leq x \vee y$ and ( $x, y)$ is codistributive $\Rightarrow$ either $\mathrm{a} \leq \mathrm{x}$ and $\mathrm{a} \leq \mathrm{y}$.

Theorem (4):- If $(x, y)$ is dually codistibutive, then for any $a \in L,(x \wedge a),(y \wedge a)$ is also dually co distributive.

Proof:- It is clear.

Theorem (5):- Relation between dually d-prime element with, (1) Distributive (2) Standard (3) Neutral

Proof:- Consider the following example for (1), i.e, dually d-prime element to distributive.

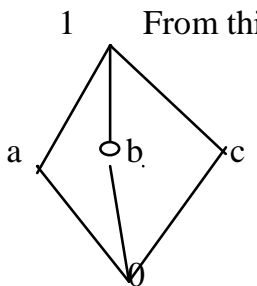

Then $a \vee(x \wedge y)=a \vee(b \wedge c)=a \vee 0=a$.

Also $(a \vee x) \wedge(a \vee y)=(a \vee b) \wedge(a \vee c)=1 \wedge 1=1$.

$$
\therefore a \neq 1 \text {. }
$$

Also $\mathrm{a} \leq \mathrm{x} \vee \mathrm{y}$

$\leq \mathrm{b} \vee \mathrm{c} \leq 1$, and $(\mathrm{x}, \mathrm{y})$ is not codistributive, also a not $\leq \mathrm{x}$ and a not $\leq \mathrm{y}$. 
Also consider the following example for (2), i.e, dually d-prime element to standard.

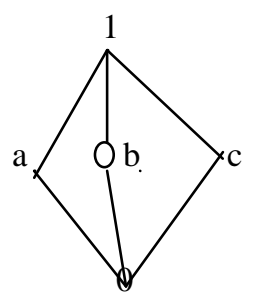

From this fig. put $\mathrm{x}=\mathrm{b}, \mathrm{y}=\mathrm{c}$

Then $x \wedge(a \vee y)=b \wedge(a \vee c)=b \wedge 1=b$.

Also $(x \wedge a) \vee(x \wedge y)=(b \wedge a) \vee(b \wedge c)=0 \vee 0=0$.

$\therefore \mathrm{b} \neq 0$.

Also $a \leq x \vee y$

$\leq \mathrm{b} \vee \mathrm{c} \leq 1$, and $(\mathrm{x}, \mathrm{y})$ is not codistributive, also a not $\leq \mathrm{x}$ and a not $\leq \mathrm{y}$.

Result (6) :- 'a' is d-prime element $\Leftrightarrow$ (a] is d-prime Ideal.

Proof:- Let 'a' be d-prime element.

Claim:- (a] is d-prime Ideal.

Let $(\mathrm{x}, \mathrm{y})$ be a co distributive pair with $\mathrm{x} \wedge \mathrm{y} \in(\mathrm{a}] . \Rightarrow \mathrm{x} \wedge \mathrm{y} \leq \mathrm{a} . \Rightarrow \mathrm{x} \leq \mathrm{a}$ or $\mathrm{y} \leq \mathrm{a}$.

If $x \leq a \Rightarrow x \in(a)$. If $y \leq a \Rightarrow y \in(a]$. Hence (a] is d-prime.

Conversely, Let (a] be d-prime.

Claim:- ' $a$ ' is d-prime element.

i.e, $a \geq x \wedge y$ and $(x, y)$ is co distributive, then $a \geq x$ or $a \geq y$.

Since $a \geq x \wedge y \Rightarrow a=a \vee(x \wedge y)=(a \vee x) \wedge(a \vee y) \Rightarrow(a \vee x) \wedge(a \vee y) \in(a]$.

Since, we know that $(\mathrm{x}, \mathrm{y})$ is co distributive then for any ' $\mathrm{a}$ ' $(\mathrm{a} \vee \mathrm{x}, \mathrm{a} \vee \mathrm{y})$ is also co distributive.

Hence $a \vee x \in(a)$ or $a \vee y \in(a]$.

If $a \vee x \in(a]$, then $a \vee x \leq a$. But $a \vee x \geq a$, hence $a \vee x=a$. Hence $x \leq a$.

If $a \vee y \in(a]$, then $a \vee y \leq a$. But $a \vee y \geq a$, hence $a \vee y=a$. Hence $y \leq a$.

Hence ' $a$ ' is d-prime element.

Result (7) :- 'a' is dually d-prime element $\Leftrightarrow$ [a) is dually d-prime and for any $(x, y)(x \wedge a),(y \wedge a)$ is also dually co distributive.

Proof:- Let 'a' be dually d-prime element $\Rightarrow a \leq x \vee y$ and ( $x, y)$ is co distributive pair $\Rightarrow a \leq x$ and $a \leq y$.

Claim:- $[a)$ is dually d-prime, where $[a)=\{x \in S / x \geq a\}$.

Let $\mathrm{x} \vee \mathrm{y} \in[\mathrm{a})$

$\Rightarrow \mathrm{a} \leq \mathrm{x} \vee \mathrm{y} \Rightarrow \mathrm{a} \leq \mathrm{x}$ and $\mathrm{a} \leq \mathrm{y}$.

If $a \leq x \Rightarrow x \in[a)$ and $a \leq y \Rightarrow y \in[a)$

Hence [a) is dually d-prime.

Conversely, let [a) is dually d-prime element.

Claim:- ' $a$ ' is dually d-prime element.

i.e, $a \leq x \vee y$ and $(x, y)$ is dually co distributive $\Rightarrow a \leq x$ and $a \leq y$.

Since $a \leq x \vee y \Rightarrow a=a \wedge(x \vee y)=(a \wedge x) \vee(a \wedge y)$.

$\Rightarrow(\mathrm{a} \wedge \mathrm{x}) \vee(\mathrm{a} \wedge \mathrm{y}) \in[\mathrm{a})$.

[ since $(\mathrm{x}, \mathrm{y})$ is dually co distributive and $(\mathrm{a} \wedge \mathrm{x}, \mathrm{a} \wedge \mathrm{y})$ is also dually co distributive]

Hence $a \wedge x \in[a)$ and $a \wedge y \in[a)$.

If $\mathrm{a} \wedge \mathrm{x} \in[\mathrm{a})$, then $\mathrm{a} \wedge \mathrm{x} \geq \mathrm{a}$, but $\mathrm{a} \wedge \mathrm{x} \leq \mathrm{a}, \therefore \mathrm{a} \wedge \mathrm{x}=\mathrm{a}$, hence $\mathrm{x} \geq \mathrm{a}$.

Also if $\mathrm{a} \wedge \mathrm{y} \in[\mathrm{a})$, then $\mathrm{a} \wedge \mathrm{y} \geq \mathrm{a}$, but $\mathrm{a} \wedge \mathrm{y} \leq \mathrm{a}, \therefore \mathrm{a} \wedge \mathrm{y}=\mathrm{a}$, hence $\mathrm{y} \geq \mathrm{a}$.

Theorem (8):- If $(a, b)$ and $(b, c)$ are dually co distributive,then $(a \wedge c, b)$ is also dually co distributive.

Proof:- Since (a,b) is dually co distributive, for any element $x \in L$, we have $(a \vee b) \wedge x=(a \wedge x) \vee(b \wedge x)$.

Also since $(b, c)$ is dually co distributive, for any element $x \in L$, we have $(b \vee c) \wedge x=(b \wedge x) \vee(c \wedge x)$.

To show that $(a \wedge c, b)$ is also dually co distributive.

Suppousing that, let $(a \wedge c, b)$ is not dually co distributive, then $[(a \wedge c) \vee b] \wedge x>[(a \wedge c) \wedge x] \vee[b \wedge x]$,for some $x \in L$, hence $\exists$ an ideal $P$ which is minimal w.r.to the property of containing $[(a \wedge c) \wedge x] \vee[b \wedge x]$ but not containing $[(\mathrm{a} \wedge \mathrm{c}) \vee \mathrm{b}] \wedge \mathrm{x}$.

Thus $[(\mathrm{a} \wedge \mathrm{c}) \wedge \mathrm{x}] \vee[\mathrm{b} \wedge \mathrm{x}] \in \mathrm{P}$ and $[(\mathrm{a} \wedge \mathrm{c}) \vee \mathrm{b}] \wedge \mathrm{x} \notin \mathrm{P}$.

Now $(\mathrm{a} \wedge \mathrm{c}) \vee \mathrm{b} \notin \mathrm{P}$ and $(\mathrm{a} \vee \mathrm{b}) \leq(\mathrm{a} \wedge \mathrm{c}) \vee \mathrm{b} \notin \mathrm{P}$. We have $(\mathrm{a} \vee \mathrm{b}) \notin \mathrm{P}$.

Similarly, $(b \vee c) \leq(a \wedge c) \vee b$, and hence $(b \vee c) \notin P$. Since, $(b \wedge x) \in P$, this shows that $b \in P$, lly $c \in P$.

If $\mathrm{a} \in \mathrm{P}$, then since $(\mathrm{a} \vee \mathrm{b}) \notin \mathrm{P}$, we have $\mathrm{b} \notin \mathrm{P}$ which is a contradiction. If $\mathrm{c} \in \mathrm{P}$, then since $(\mathrm{b} \vee c) \notin \mathrm{P}$,

We have $\mathrm{b} \notin \mathrm{P}$, which is also a contradiction. Hence $(\mathrm{a} \wedge \mathrm{c}, \mathrm{b})$ is dually co distributive.

Theorem (9):- Suppouse $I$ is a sublattice of $L$ and $m_{a}, a \in I$ and $m_{a}$ is an Ideal of $I$, minimal w.r.to the property of containing ' $a$ ', then there is a d-prime ideal $\mathrm{P}$ of $\mathrm{L} \ni \mathrm{P} \cap \mathrm{I}=\mathrm{m}_{\mathrm{a}}$. 
Proof:- Let $\mathrm{x} \in\left[\mathrm{m}_{\mathrm{a}}\right) \cap(\mathrm{a}]$, then $\mathrm{x} \in\left[\mathrm{m}_{\mathrm{a}}\right)$ and $\mathrm{x} \in(\mathrm{a}]$, so that $\mathrm{x} \leq \mathrm{a}$ for some $\mathrm{x} \in\left[\mathrm{m}_{\mathrm{a}}\right)$ and hence $\mathrm{x} \geq \mathrm{k}$ for some $\mathrm{k} \in \mathrm{m}_{\mathrm{a}}$. Thus $\mathrm{a} \geq \mathrm{x} \geq \mathrm{k} \Rightarrow \mathrm{a} \geq \mathrm{k}$ for some $\mathrm{k} \in \mathrm{m}_{\mathrm{a}}$ which is a contradiction.

Hence $\left[\mathrm{m}_{\mathrm{a}}\right) \cap(\mathrm{a}]=\varnothing . \therefore \exists \mathrm{a}$ d-prime ideal $\mathrm{P}$ of $\mathrm{L} \ni\left[\mathrm{m}_{\mathrm{a}}\right) \subseteq \mathrm{P}$, and $\mathrm{P} \cap(\mathrm{a}]=\varnothing$, hence 'P' is d-prime. Claim:- $\mathrm{P} \cap \mathrm{I}=\mathrm{m}_{\mathrm{a}}$

Now $\mathrm{m}_{\mathrm{a}} \subseteq \mathrm{P}, \mathrm{m}_{\mathrm{a}} \subseteq \mathrm{I} \Rightarrow \mathrm{m}_{\mathrm{a}} \subseteq \mathrm{P} \cap \mathrm{I}$

Suppouse $\mathrm{x} \in \mathrm{P} \cap \mathrm{I}$ so that $\mathrm{x} \in \mathrm{P}$ and $\mathrm{x} \in \mathrm{I}$,

If $\mathrm{x} \notin \mathrm{m}_{\mathrm{a}}$ then $\mathrm{a} \in \mathrm{m}_{\mathrm{a}} \wedge[\mathrm{x})$ and hence $\mathrm{a} \geq \mathrm{k} \wedge \mathrm{x}$, for some $\mathrm{k} \in \mathrm{m}_{\mathrm{a}}$.

$\therefore \mathrm{k} \in \mathrm{m}_{\mathrm{a}} \Rightarrow \mathrm{k} \in \mathrm{P}$. for some $\mathrm{x} \in \mathrm{P}$. since $\mathrm{k} \in \mathrm{P}, \mathrm{x} \wedge \mathrm{k} \in \mathrm{P}$ so that, $\mathrm{a} \notin \mathrm{P}$ which is a contradiction.

Hence $\mathrm{x} \in \mathrm{m}_{\mathrm{a}}$.

Thus $\mathrm{P} \cap \mathrm{I} \subseteq \mathrm{m}_{\mathrm{a}}$----------------(**)

From $(*)$ and $(* *), \quad \mathrm{P} \cap \mathrm{I}=\mathrm{m}_{\mathrm{a}}$.

Lemma (10):- If $\mathrm{L}$ is any lattice then every dually d-meet irreducible element is dually d-prime.

Proof:- Proof is clear.

References

[1]. Gratzer,G.(1978) : "General Lattice Theory", Akademic Press, New York.

[2]. Sazsaz,Gabor(1963) : "Introduction to Lattice Theory" Akademic Press, New York and London.

[3]. Srinivas,K.V.R.(2005) : “On D-Prime Ideals of a Lattice” Bull.Cal.Math.Soc.,97(1) 79-94(2005). 